\title{
Sífilis em jovens conscritos brasileiros, 2016: aspectos sociodemográficos, comportamentais e clínicos
}

\section{Syphilis in young Brazilian military recruits in 2016: sociodemographic, behavioral, and clinical aspects}

\section{Sífilis en jóvenes reclutas brasileños, 2016: aspectos sociodemográficos, comportamentales y clínicos}

Francisca Lidiane Sampaio Freitas 1,2 Ximena Pamela Díaz Bermúdez 3

Edgar Merchan-Hamann 3

Leonardo Rapone da Motta 4

Machline Paim Paganella 4

Rosa Dea Sperhacke 4

Gerson Fernando Mendes Pereira 2

\section{Resumo}

O objetivo foi caracterizar aspectos sociodemográficos, comportamentais e clinicos entre conscritos brasileiros, segundo a prevalência de sífilis. Estudo descritivo desenvolvido valendo-se de pesquisa nacional de base populacional, realizada em 2016, com amostra probabilística de jovens conscritos de 17 a 22 anos de idade. Realizou-se autoaplicação de questionários confidenciais. As amostras de sangue total dos participantes foram coletadas para testagem de sífilis, com a utilização de testes treponêmicos e não treponêmicos. Empregaram-se técnicas de estatística descritiva para estimar as prevalências de sifilis e a distribuição de frequências entre as variáveis pesquisadas, considerando os intervalos de 95\% de confiança (IC95\%), após a ponderação dos dados. Do total de 37.282 participantes, 73,7\% haviam iniciado a vida sexual. As prevalências de sifilis na vida e de sifilis confirmada foram de 1,6\% e 1,1\%, respectivamente. As seguintes variáveis populacionais apresentaram maior prevalência de sífilis: ausência de acesso à Internet no domicílio; início da atividade sexual antes dos 14 anos; categoria de exposição homens que fazem sexo com homens; práticas sexuais com mais de cinco parcerias; recebimento de presentes, drogas ou outros incentivos em troca de sexo; e história prévia de sintomatologia de infecções sexualmente transmissiveis. Observou-se o aumento de sífilis entre os jovens conscritos brasileiros, em comparação aos inquéritos anteriores. Tal incremento reforça a importância dessa população sentinela para realizar vigilância ativa, de forma a subsidiar estratégias de atenção à saúde dos jovens, incluindo cenários escolares.

Prevalência; Inquéritos Epidemiológicos; Sífilis

\author{
Correspondência \\ F. L. S. Freitas \\ Programa de Pós-graduação em Saúde Coletiva, Universidade \\ de Brasília. \\ Campus Universitário Darcy Ribeiro s/n, Brasília, DF \\ 70910-900, Brasília. \\ lidianesfreitas18@gmail.com \\ 1 Programa de Pós-graduação em Saúde Coletiva, Universidade \\ de Brasília, Brasília, Brasil. \\ 2 Secretaria de Vigilância em Saúde, Ministério da Saúde, \\ Brasília, Brasil. \\ 3 Faculdade de Ciências da Saúde, Universidade de Brasília, \\ Brasília, Brasil. \\ 4 Instituto de Pesquisas em Saúde, Universidade de Caxias do \\ Sul, Caxias do Sul, Brasil.
}




\section{Introdução}

A sífilis é uma infecção sexualmente transmissível (IST) reemergente, que persiste como desafio na saúde pública global, com o aumento do número de casos na última década 1,2. No Brasil, observou-se que a taxa de detecção da sífilis adquirida aumentou de 34,1 casos por 100 mil habitantes, em 2015, para 75,8 casos por 100 mil habitantes, em 2018, de acordo com o Sistema de Informação de Agravos de Notificação (SINAN). Em 2018, cerca de 46\% dos casos de sífilis adquirida pertenciam à faixa etária de 13 a 29 anos, com maior tendência de aumento na população de 20 a 29 anos 3 .

Os inquéritos em saúde pública realizados periodicamente no país, como o estudo de base populacional com amostra de conscritos das Forças Armadas, complementam e atualizam os dados programáticos sobre as IST. Esses jovens apresentam diferentes perfis sociodemográficos no conjunto do território nacional 4 e compõem uma faixa etária caracterizada por importantes mudanças biopsicossociais e comportamentais, com diversos riscos para a saúde 5 .

O primeiro estudo de abrangência nacional com jovens conscritos foi realizado em 1996 e abordou o conhecimento sobre a transmissão do HIV. Nas edições subsequentes (1997-2000, 2002 e 2007), incluíram-se tópicos acerca das IST e hepatites virais, práticas sexuais, uso de álcool e outras drogas, bem como marcadores biológicos para a estimativa da prevalência de HIV e sífilis 4,6. Nas pesquisas conduzidas em 2002 e 2007, a prevalência de sífilis diminuiu de $0,8 \%$ para $0,5 \%$, respectivamente 6,7 .

Em 2016, foi realizada a oitava edição dessa série de investigações, a qual subsidiou a presente análise, que tem por objetivo estimar a prevalência de sífilis e sua distribuição, segundo variáveis sociodemográficas, comportamentais e clínicas entre conscritos.

\section{Métodos}

\section{População do estudo e amostragem}

Esta análise faz um recorte descritivo do estudo transversal realizado de agosto a dezembro de 2016, com amostra probabilística de jovens entre 17 e 22 anos de idade, que foram chamados para comissões de seleção, após a fase de alistamento, com vistas ao serviço militar obrigatório. Tal pesquisa é resultado de cooperação técnica entre o Ministério da Saúde e o Ministério da Defesa, desde a década de 19908.

O cálculo amostral teve como base a estimativa de $0,12 \%$ da prevalência de infecção pelo HIV em homens jovens, no ano de 2007 6, considerando o intervalo de 95\% de confiança (IC95\%) e o erro bicaudal de $0,04 \%$. O tamanho da amostra foi calculado em aproximadamente 40 mil jovens, o que representa cerca de $6 \%$ dos conscritos 9 .

A amostragem usou conglomerados em dois estágios: (i) definição de 87 comissões de seleção com representação nos 26 estados brasileiros e no Distrito Federal; e (ii) definição do total de conscritos, conforme o tamanho de cada comissão 9 . Foram excluídos da amostra os analfabetos; os indivíduos com ausência de informação sobre idade, grau de escolaridade e município de origem; e aqueles que se recusaram a assinar o Termo de Consentimento Livre e Esclarecido (TCLE).

\section{Coleta de dados e análise laboratorial}

Os dados sociodemográficos foram coletados por meio de um questionário confidencial e autoaplicável, contendo 74 perguntas sobre características sociais e populacionais, práticas de comportamento sexual, problemas relacionados às IST e uso de drogas lícitas/ilícitas. Tais questionários foram processados no Laboratório de Pesquisa em HIV/Aids da Universidade de Caxias do Sul, por meio da utilização do OpenText TeleForm 11.1 (https://www.opentext.com/) 8 .

Com base em uma única coleta de amostra de sangue total de cada participante, utilizou-se a seguinte sequência para a testagem de sífilis: (i) teste inicial (T1) - ensaio treponêmico automatizado, com método de quimioluminescência (Architect Syphilis TP, da Abbott Laboratories, Wiesbaden, Alemanha); (ii) teste complementar (T2) - ensaio não treponêmico (VDRL, da Wiener Lab., Rosario, Argentina), realizado em caso de ensaio inicial reagente; e (iii) teste treponêmico (T3) (FTA-Abs, da 
Wama Diagnóstica, São Carlos, Brasil) no caso de resultados discordantes, em que o T1 apresentouse reagente e o T2 não reagente. A testagem de sífilis foi realizada no Laboratório Central (Lacen) de Vespasiano (Minas Gerais), em conformidade com os critérios técnicos dos fabricantes 8 .

A sífilis confirmada foi definida pela positividade de T1 e T2 ou T1 e T3, e a sífilis na vida foi considerada em caso de $\mathrm{T} 1$ reagente. Os jovens que tiveram resultado reagente foram orientados a comparecer a unidades de saúde pública para acompanhamento clínico e laboratorial.

\section{Variáveis do estudo}

As variáveis sociodemográficas analisadas foram: região de residência; idade; raça/cor; estado civil/ conjugal; escolaridade; e acesso à Internet no domicílio.

A respeito do conhecimento sobre IST, foram verificadas: forma de transmissão; presença de sinais e sintomas; existência de tratamento; e disponibilidade gratuita de testes de HIV, sífilis e hepatites virais.

Para a análise das práticas sexuais, foram estudadas: idade da primeira relação sexual; uso de preservativo na primeira e na última relação sexual; ter tido relação sexual com outros homens (homens que fazem sexo com homens - HSH), independentemente das relações com mulheres; número de parcerias sexuais no mês anterior à aplicação do questionário; e recebimento de presentes, drogas ou outros incentivos para ter relação sexual nos 12 meses anteriores ao estudo.

Em relação a antecedentes de IST e busca de tratamento, as variáveis consideradas foram: história prévia de pelo menos um sinal/sintoma de IST; história prévia de sinais e sintomas de IST; e busca e realização do tratamento.

\section{Análise estatística}

O software estatístico utilizado foi o SPSS 20.0 (https://www.ibm.com/), empregando-se o módulo de amostras complexas. Em decorrência da complexidade do plano amostral, usou-se a ponderação das medidas, que considera um fator de expansão que é o inverso do produto das probabilidades de seleção em cada estágio, seguido da razão entre a população de 18 anos e o tamanho da amostra ponderada.

A análise levou em conta a estratégia de amostragem complexa que considerou probabilidades distintas de seleção, estratificação e efeito de conglomeração das unidades, calibração para não-resposta e demais ajustes 10. Tal procedimento de calibração foi aplicado segundo a distribuição do censo por tamanho da população da cidade de residência, idade e nível de escolaridade 8 .

Nesta análise, foram aplicadas técnicas de estatística descritiva para a obtenção de estimativa das prevalências de sífilis e distribuição de frequências entre as variáveis estudadas. A estimativa das prevalências de sífilis foi calculada para cada grupo, por meio de proporções. O numerador correspondeu ao número de casos de sífilis confirmada com determinada característica, ao passo que o denominador correspondeu ao total de participantes com esta mesma característica, considerando IC95\%, após a ponderação.

\section{Aspectos éticos}

A participação dos conscritos aconteceu de forma voluntária, por meio de assinatura do TCLE. O estudo foi aprovado pela Comissão Nacional de Ética em Pesquisa (CONEP; parecer no 278.616), e pelo Comitê de Ética em Pesquisa (CEP) da Universidade de Caxias do Sul (parecer no 1.074.338/2015, atualizado para o parecer no 1.422.093/2016). 


\section{Resultados}

Baseando-se no cálculo do tamanho amostral, estabelecido em aproximadamente 40 mil conscritos, o estudo atingiu inicialmente o total de 38.247 participantes. Desses, 965 foram excluídos devido à ausência de informação relacionada à idade, grau de escolaridade e município de origem, entre outros. A amostra final incluiu 37.282 participantes $(93,2 \%)$ do universo inicialmente calculado.

A prevalência de sífilis na vida foi de 1,6\% (IC95\%: 1,33-1,98). A Tabela 1 mostra que a prevalência de sífilis confirmada foi de 1,1\% (IC95\%: 0,85-1,40). Embora uma proporção menor de jovens $(1,4 \%)$ tivesse 21 anos, estes apresentaram maior prevalência de sífilis (4,9\%; IC95\%: 2,02-11,78) em relação às demais faixas etárias, sem sobreposição deste IC95\% aos parâmetros das três primeiras categorias de idade (17 a 19 anos).

A maior parte da amostra $(76,2 \%)$ residia nas regiões Sudeste, Nordeste e Centro-oeste, com prevalências de sífilis menores, já nas regiões Norte e Sul as prevalências foram maiores, sobrepondo-se os respectivos IC95\%.

As maiores prevalências de sífilis ocorreram entre pretos $(1,7 \%)$ e pardos $(1,1 \%)$, que somaram $58,1 \%$ do total de conscritos. A menor prevalência $(0,5 \%)$ foi verificada entre indígenas, os quais representaram 1,7\% da amostra, com sobreposição dos IC95\%.

Quanto ao estado civil/conjugal, 97,7\% dos jovens afirmaram ser solteiros; entre estes, a prevalência de sífilis foi de $1,1 \%$. Por outro lado, as prevalências foram mais altas entre os casados (2\%) e os divorciados (3,3\%), que representaram apenas 1,7\% da amostra, com sobreposição dos IC95\%.

A proporção dos participantes com maior escolaridade (Ensino Médio completo ou Superior completo/incompleto), que totalizou $50,7 \%$ da amostra, apresentou prevalências de sífilis menores, oscilando entre $0,2 \%$ e $0,8 \%$, ao passo que, nas categorias de menor escolaridade, as prevalências foram maiores e variaram entre 1,2\% e 2,9\%. No entanto, há sobreposição entre os IC95\% de todas as categorias.

Verifica-se, ainda, que 69,8\% dos conscritos referiram ter acesso à Internet no domicílio, sendo menor a prevalência de sífilis entre estes $(0,7 \%)$ em comparação àqueles sem acesso à Internet, que registraram prevalência mais alta (2\%). Nesse caso, não foi observada sobreposição dos IC95\%.

A Tabela 2 indica que 93,6\% dos conscritos apresentaram conhecimento incorreto em relação às formas de transmissão das IST. Foi interessante constatar que 43,3\% dos conscritos concordaram que se pode contrair IST comendo alimentos contaminados, por meio de banho em rios ou praias $(67,1 \%)$, picada de mosquito (58,9\%), banheiros públicos (62,4\%), ou beijo (57,3\%). No entanto, $95,2 \%$ sabem que uma IST pode ser transmitida por relação sexual sem o uso do preservativo e 64,5\% acreditam que o sexo oral é a forma de transmissão. Ressalta-se também que $24 \%$ do total de jovens acreditam que uma pessoa pode não ter sintomatologia, mesmo estando doente; $47,2 \%$ reconhecem que a úlcera anogenital é sinal de IST; e 34,6\% não sabem o que o homem apresenta quando tem uma IST.

A grande maioria $(84,9 \%)$ concorda que existe tratamento para pessoas com IST. Entretanto, apenas $25,2 \%$ e $38,1 \%$ dos conscritos sabem onde são ofertados testes gratuitos de sífilis e HIV, respectivamente. Nesses casos, também houve sobreposição dos IC95\% referentes às prevalências de sífilis.

A maioria dos jovens gostaria de obter informações acerca das IST por meio da televisão $(71,8 \%)$, da internet $(69,4 \%)$ e da escola $(68,9 \%)$, não havendo diferença nas prevalências de sífilis entre estas categorias (dados não apresentados na tabela).

A maior parte dos conscritos (73,7\%) declarou ter mantido relação sexual (oral, vaginal ou anal) alguma vez na vida. Desses jovens, 16,2\% afirmaram ter iniciado a atividade sexual antes dos 14 anos de idade, entre os quais foi estimada maior prevalência de sífilis $(2,8 \%)$ em relação aos que iniciaram a vida sexual com 14 anos ou mais, que apresentaram menor prevalência (1\%), ressaltando-se que não houve sobreposição dos respectivos IC $95 \%$. Cerca de $41 \%$ dos jovens indicaram ter tido mais de cinco parcerias sexuais no mês anterior à pesquisa, sendo que, entre estes, a prevalência de sífilis foi maior $(2,1 \%)$ do que a dos que indicaram até cinco parcerias, sem sobreposição dos IC95\% (Tabela 3).

Independentemente do tipo de parceria, $60 \%$ dos jovens referiram ter usado preservativo na primeira e na última relação sexual, sem diferença nas prevalências de sífilis entre as duas categorias. Outrossim, destaca-se que 4,4\% dos conscritos afirmaram ter relação sexual com outros homens, apresentando maior prevalência de sífilis $(4,6 \%$; IC95\%: 2,73-7,62) em relação aos que não eram HSH, sem sobreposição entre os respectivos IC95\%. 
Tabela 1

Características sociodemográficas, segundo prevalência de sífilis confirmada entre conscritos. Brasil, 2016.

\begin{tabular}{|c|c|c|c|}
\hline \multirow[t]{2}{*}{ Variável } & \multirow[t]{2}{*}{ n (\%) } & \multicolumn{2}{|c|}{ Prevalência de sífilis } \\
\hline & & n (\%) & IC95\% \\
\hline Total & $37.282(100,00)$ & $393(1,09)$ & $0,85-1,40$ \\
\hline \multicolumn{4}{|l|}{ Idade (anos) } \\
\hline 17 & $6.944(18,62)$ & $52(0,78)$ & $0,41-1,48$ \\
\hline 18 & $24.374(65,38)$ & $267(1,14)$ & $0,81-1,60$ \\
\hline 19 & $3.887(10,43)$ & $30(0,79)$ & $0,54-1,16$ \\
\hline 20 & $1.320(3,54)$ & $16(1,26)$ & $0,60-2,63$ \\
\hline 21 & $514(1,38)$ & $25(4,99)$ & $2,02-11,78$ \\
\hline 22 & $243(0,65)$ & $16(1,32)$ & $0,59-2,91$ \\
\hline \multicolumn{4}{|l|}{ Região de residência } \\
\hline Centro-oeste & $2.841(7,62)$ & $19(0,67)$ & $0,36-1,24$ \\
\hline Nordeste & $10.969(29,42)$ & $104(0,97)$ & $0,52-1,80$ \\
\hline Norte & $3.683(9,88)$ & $43(1,28)$ & $0,76-2,17$ \\
\hline Sudeste & $14.614(39,20)$ & $154(1,09)$ & $0,74-1,60$ \\
\hline Sul & $5.176(13,88)$ & $73(1,48)$ & $1,03-2,12$ \\
\hline \multicolumn{4}{|l|}{ Raça/Cor } \\
\hline Branca & $13.365(35,85)$ & $119(0,92)$ & $0,53-1,59$ \\
\hline Preta & $5.842(15,67)$ & $98(1,73)$ & $0,96-3,08$ \\
\hline Amarela & $1.487(3,99)$ & $13(0,87)$ & $0,38-1,96$ \\
\hline Parda & $15.821(42,43)$ & $160(1,05)$ & $0,74-1,50$ \\
\hline Indígena & $618(1,66)$ & $3(0,53)$ & $0,23-1,18$ \\
\hline Não respondeu & $150(0,40)$ & - & - \\
\hline \multicolumn{4}{|l|}{ Estado civil/conjugal } \\
\hline Casado & $625(1,68)$ & $12(2,03)$ & $1,00-4,24$ \\
\hline Divorciado & $16(0,04)$ & $0,48(3,35)$ & $0,45-20,92$ \\
\hline Solteiro & $36.436(97,73)$ & $379(1,08)$ & $0,83-1,39$ \\
\hline Desquitado/Separado judicialmente/Viúvo & $41(0,11)$ & $0(0,00)$ & - \\
\hline Sem informação & $163(0,44)$ & - & - \\
\hline \multicolumn{4}{|l|}{ Escolaridade } \\
\hline Ensino Fundamental incompleto & $2.422(6,50)$ & $28(1,21)$ & $0,79-1,84$ \\
\hline Ensino Fundamental completo & $2.866(7,69)$ & $81(2,88)$ & $1,38-5,90$ \\
\hline Ensino Médio e/ou Técnico incompleto & $13.086(35,10)$ & $157(1,24)$ & $0,76-2,04$ \\
\hline Ensino Médio e/ou Técnico completo & $12.201(32,73)$ & $97(0,83)$ & $0,48-1,42$ \\
\hline Superior incompleto & $5.017(13,46)$ & $27(0,57)$ & $0,22-1,46$ \\
\hline Superior completo & $1.691(4,54)$ & $3(0,20)$ & $0,09-0,49$ \\
\hline \multicolumn{4}{|l|}{ Acesso à Internet no domicílio } \\
\hline Sim & $26.009(69,76)$ & $174(0,69)$ & $0,50-0,95$ \\
\hline Não & $11.273(30,24)$ & $220(2,03)$ & $1,38-2,98$ \\
\hline
\end{tabular}

IC95\%: intervalo de 95\% de confiança.

Apenas 2\% relataram ter recebido presentes, drogas ou outros incentivos para ter relação sexual nos 12 meses anteriores ao estudo, destacando-se com maior prevalência de sífilis $(3,5 \%)$ em comparação aos que não receberam incentivos, sem sobreposição entre os IC95\%. Por outro lado, 2,7\% dos participantes pagaram para manter relação sexual nos 12 meses prévios à pesquisa, não divergindo a prevalência de sífilis destes em relação à daqueles que não pagaram por sexo. 
Tabela 2

Conhecimento sobre infecções sexualmente transmissíveis (IST), segundo prevalência de sífilis confirmada entre conscritos. Brasil, 2016.

\begin{tabular}{|c|c|c|c|}
\hline \multirow[t]{2}{*}{ Variável } & \multirow[t]{2}{*}{ n (\%) } & \multicolumn{2}{|c|}{ Prevalência de sífilis } \\
\hline & & n (\%) & IC95\% \\
\hline \multicolumn{4}{|l|}{ Conhecimento correto sobre transmissão das IST } \\
\hline $\operatorname{sim}$ & $2.389(6,41)$ & $26(1,12)$ & $0,46-2,71$ \\
\hline Não & $34.893(93,59)$ & $367(1,09)$ & $0,85-1,40$ \\
\hline \multicolumn{4}{|l|}{ Conhecimento sobre a transmissão das IST } \\
\hline \multicolumn{4}{|l|}{ Concordou } \\
\hline Compartilhamento de agulhas e seringas & $33.538(89,96)$ & $318(0,98)$ & $0,75-1,29$ \\
\hline Relação sexual sem preservativo & $35.477(95,16)$ & $332(0,97)$ & $0,75-1,25$ \\
\hline Durante a gravidez, parto e amamentação & $25.212(67,63)$ & $244(1,00)$ & $0,73-1,38$ \\
\hline Sexo oral & $24.066(64,55)$ & $271(1,17)$ & $0,86-1,59$ \\
\hline \multicolumn{4}{|l|}{ Discordou } \\
\hline Comendo alimentos contaminados & $21.126(56,67)$ & $199(0,98)$ & $0,69-1,40$ \\
\hline Banho em rios ou praias & $25.017(67,10)$ & $235(0,97)$ & $0,69-1,37$ \\
\hline Picada de mosquito & $15.326(41,11)$ & $121(0,82)$ & $0,54-1,24$ \\
\hline Banheiros públicos & $14.023(37,61)$ & $157(1,15)$ & $0,71-1,87$ \\
\hline Beijo & $15.918(42,70)$ & $107(0,70)$ & $0,41-1,18$ \\
\hline \multicolumn{4}{|l|}{ Conhecimento sobre a transmissão vertical das IST } \\
\hline Gonorreia & $5.610(15,05)$ & $92(1,70)$ & $1,00-2,87$ \\
\hline Sífilis & $7.962(21,36)$ & $106(1,38)$ & $0,98-1,96$ \\
\hline HPV & $7.761(20,82)$ & $59(0,78)$ & $0,48-1,27$ \\
\hline Herpes genital & $6.380(17,11)$ & $55(0,89)$ & $0,53-1,49$ \\
\hline HIV/aids & $25.150(67,46)$ & $248(1,02)$ & $0,74-1,40$ \\
\hline Hepatite & $17.248(46,26)$ & $182(1,09)$ & $0,74-1,60$ \\
\hline Todas as mencionadas & $6.202(16,64)$ & $71(1,19)$ & $0,62-2,25$ \\
\hline Nenhuma & $2.501(6,71)$ & $15(0,64)$ & $0,23-1,72$ \\
\hline \multicolumn{4}{|l|}{ Conhecimento sobre sinais e sintomas das IST } \\
\hline Nada, mesmo estando doente & $8.959(24,03)$ & $105(1,21)$ & $0,65-2,24$ \\
\hline Verruga anogenital & $12.496(33,52)$ & $147(1,21)$ & $0,83-1,78$ \\
\hline Bolhas no pênis & $14.476(38,83)$ & $186(1,33)$ & $0,88-2,00$ \\
\hline Úlcera anogenital & $17.607(47,23)$ & $207(122)$ & $0,83-1,79$ \\
\hline Corrimento uretral & $14.412(38,66)$ & $163(1,17)$ & $0,81-1,68$ \\
\hline Disúria & $14.386(38,59)$ & $162(1,16)$ & $0,76-1,78$ \\
\hline Não sabe & $12.888(34,57)$ & $88(0,71)$ & $0,42-1,19$ \\
\hline \multicolumn{4}{|l|}{ Existe tratamento para pessoas com IST } \\
\hline Concorda & $31.671(84,95)$ & $322(1,05)$ & $0,80-1,38$ \\
\hline Discorda & $1.202(3,22)$ & $5(0,46)$ & $0,20-1,05$ \\
\hline Não sabe & $4.409(11,83)$ & $66(1,57)$ & $0,67-3,63$ \\
\hline \multicolumn{4}{|l|}{ Sabe onde há testes gratuitos } \\
\hline HIV & $14.209(38,11)$ & $236(1,72)$ & $1,25-2,35$ \\
\hline Sífilis & $9.390(25,19)$ & $197(2,18)$ & $1,47-3,23$ \\
\hline Hepatites & $12.606(33,81)$ & $197(1,62)$ & $1,10-2,39$ \\
\hline
\end{tabular}

IC95\%: intervalo de 95\% de confiança.

Nota: $\mathrm{N}$ total $=37.282$ 
Tabela 3

Comportamento e práticas sexuais entre conscritos que iniciaram a vida sexual, segundo prevalência de sífilis confirmada. Brasil, 2016.

\begin{tabular}{|c|c|c|c|}
\hline \multirow[t]{2}{*}{ Variável } & \multirow[t]{2}{*}{ n (\%) } & \multicolumn{2}{|c|}{ Prevalência de sífilis } \\
\hline & & n (\%) & IC95\% \\
\hline \multicolumn{4}{|c|}{ Idade da primeira relação sexual (anos) } \\
\hline$<14$ & $4.719(16,18)$ & $129(2,84)$ & $1,71-4,67$ \\
\hline$>14$ & $23.964(82,16)$ & $228(0,98)$ & $0,68-1,41$ \\
\hline Não respondeu & $486(1,66)$ & $3(0,55)$ & $0,21-1,46$ \\
\hline \multicolumn{4}{|c|}{ Número de parcerias sexuais no último mês } \\
\hline Até 5 & $17.296(59,20)$ & $114(0,69)$ & $0,37-1,27$ \\
\hline Mais de 5 & $11.934(40,80)$ & $245(2,11)$ & $1,53-2,90$ \\
\hline \multicolumn{4}{|c|}{ Uso de preservativo na primeira relação sexual } \\
\hline Sim & $17.885(60,44)$ & $194(1,12)$ & $0,75-1,68$ \\
\hline Não & $9.916(33,51)$ & $149(1,56)$ & $1,03-2,35$ \\
\hline Não se lembra & $1.550(5,24)$ & $16(1,04)$ & $0,56-1,91$ \\
\hline Não respondeu & $241(0,81)$ & $3(1,18)$ & $0,42-3,27$ \\
\hline \multicolumn{4}{|c|}{ Uso de preservativo na última relação sexual } \\
\hline Sim & $17.633(59,99)$ & $190(1,11)$ & $0,76-1,63$ \\
\hline Não & $10.629(36,16)$ & $151(1,47)$ & $0,93-2,34$ \\
\hline Não se lembra & $791(2,69)$ & $17(2,19)$ & $0,65-7,17$ \\
\hline Não respondeu & $340(1,16)$ & $2(0,56)$ & $0,17-1,81$ \\
\hline \multicolumn{4}{|l|}{$\mathrm{HSH}$} \\
\hline Sim & $1.308(4,41)$ & $59(4,59)$ & $2,73-7,62$ \\
\hline Não & $28.208(95,08)$ & $301(1,11)$ & $0,80-1,52$ \\
\hline Outros & $49(0,16)$ & $0(0,00)$ & - \\
\hline Não respondeu & $105(0,35)$ & $1(1,07)$ & $0,36-3,13$ \\
\hline \multicolumn{4}{|c|}{ Recebeu presentes, drogas ou outros incentivos para ter relação sexual } \\
\hline \multicolumn{4}{|c|}{ nos últimos 12 meses } \\
\hline Sim & $578(1,96)$ & $20(3,49)$ & $1,68-7,10$ \\
\hline Não & $27.889(94,44)$ & $334(1,24)$ & $0,93-1,65$ \\
\hline Não sabe & $267(0,90)$ & $2(0,76)$ & $0,30-1,92$ \\
\hline Não respondeu & $798(2,70)$ & $5(0,61)$ & $0,31-1,23$ \\
\hline
\end{tabular}

HSH: homens que fazem sexo com homens; IC95\%: intervalo de 95\% de confiança.

Nota: $\mathrm{N}$ total $=37.282$.

$\mathrm{Na}$ Tabela 4, 20,3\% dos jovens apresentaram história de pelo menos um sinal ou sintoma de IST em algum momento da vida, estimando-se, entre estes, maior prevalência de sífilis $(2,7 \%)$. Em contrapartida, entre os que não mencionaram antecedentes de IST a prevalência foi menor $(0,7 \%)$, sem coincidirem os IC95\% das duas categorias.

Observa-se que 3,6\% declararam história prévia de úlcera anogenital. Nesses, a prevalência de sífilis foi maior $(9,3 \%)$ do que a das demais categorias de sintomatologia das IST, exceto em relação às verrugas, sem sobreposição dos respectivos IC95\%.

Entre aqueles com história de pelo menos um sinal ou sintoma de IST, observou-se que 39\% procuraram serviços de saúde públicos ou particulares; 9,9\% não procuraram ajuda; e 3,5\% não buscaram tratamento porque os sinais/sintomas desapareceram. Nessas categorias, a prevalência de sífilis variou entre 1,4\% e 2,9\%, com sobreposição dos respectivos IC95\%. Entretanto, um percentual menor de jovens $(1,4 \%)$ buscou tratamento junto ao balconista da farmácia, mas apresentou maior prevalência de sífilis (4\%; IC95\%: 1,01-14,57), com sobreposição ao IC95\% das demais categorias.

Destaca-se ainda que, entre os que tiveram história prévia de pelo menos um sinal ou sintoma de IST, 33,5\% realizaram o tratamento e apresentaram prevalência de sífilis maior (3,5\%) em relação àqueles que não realizaram a terapêutica medicamentosa, sobrepondo-se ao IC95\% destes últimos. 
Tabela 4

História prévia de infecções sexualmente transmissíveis (IST) e busca de tratamento entre conscritos, segundo prevalência de sífilis confirmada. Brasil, 2016.

\begin{tabular}{|c|c|c|c|}
\hline \multirow[t]{2}{*}{ Variável } & \multirow[t]{2}{*}{ n (\%) } & \multicolumn{2}{|c|}{ Prevalência de sífilis } \\
\hline & & n (\%) & IC95\% \\
\hline \multicolumn{4}{|l|}{ História prévia de pelo menos um sinal/sintoma de IST } \\
\hline Sim & $7.572(20,31)$ & $203(2,74)$ & $1,83-4,10$ \\
\hline Não & $29.710(79,69)$ & $191(0,67)$ & $0,49-0,91$ \\
\hline \multicolumn{4}{|l|}{ História prévia de sinais/sintomas de IST } \\
\hline Verruga anogenital & $1.006(2,70)$ & $50(5,09)$ & $1,80-13,55$ \\
\hline Bolhas no pênis & $756(2,03)$ & $14(1,83)$ & $1,04-3,18$ \\
\hline Úlcera anogenital & $1.342(3,60)$ & $124(9,35)$ & $5,42-15,67$ \\
\hline Corrimento uretral & $488(1,31)$ & $14(2,82)$ & $1,69-4,66$ \\
\hline Disúria & $2.685(7,20)$ & $37(1,41)$ & $0,55-3,59$ \\
\hline Nenhum & $28.033(75,19)$ & $168(0,62)$ & $0,45-0,86$ \\
\hline \multicolumn{4}{|l|}{ Busca de tratamento * } \\
\hline Serviço de saúde pública ou particular & $2.948(38,94)$ & $93(3,20)$ & $1,79-5,65$ \\
\hline Balconista da farmácia & $519(1,39)$ & $21(4,01)$ & $1,01-14,57$ \\
\hline Família/amigos & $2.827(7,58)$ & $71(2,54)$ & $1,18-5,40$ \\
\hline Não procurou ajuda & $3.681(9,87)$ & $50(1,42)$ & $0,69-2,91$ \\
\hline Problema desapareceu sem buscar tratamento & $1.307(3,51)$ & $37(2,88)$ & $0,66-11,65$ \\
\hline Outros & $193(0,52)$ & $3(1,38)$ & $0,44-4,24$ \\
\hline \multicolumn{4}{|l|}{ Realização de tratamento * } \\
\hline Sim & $4.020(33,51)$ & $138(3,51)$ & $2,05-5,95$ \\
\hline Não & $5.167(43,08)$ & $118(2,35)$ & $1,27-4,29$ \\
\hline Não se lembra & $2.404(20,05)$ & $27(1,14)$ & $0,50-2,61$ \\
\hline Não respondeu & $403(3,36)$ & $5(1,23)$ & $0,49-3,04$ \\
\hline
\end{tabular}

IC95\%: intervalo de 95\% de confiança.

Nota: $\mathrm{N}$ total $=37.282$

* Percentuais calculados em relação àqueles que tiveram pelo menos um sinal/sintoma de IST.

\section{Discussão}

Os resultados revelaram a prevalência de sífilis na vida $(1,6 \%)$ e sífilis confirmada $(1,1 \%)$. A distribuição da sífilis confirmada manifestou maior prevalência nas variáveis e categorias cujos IC95\% não se sobrepunham entre si: ausência de acesso à Internet no domicílio; início da atividade sexual antes dos 14 anos; categoria de exposição HSH; práticas sexuais com mais de cinco parcerias; recebimento de presentes, drogas ou outros incentivos em troca de sexo; e história prévia de sintomatologia de IST.

No Brasil, em 2016, a prevalência de sífilis na vida triplicou em relação ao estudo dos conscritos de 2007 6,7. Dada a mesma comparação com o inquérito nacional de 2007 7, a prevalência de sífilis na vida aumentou cerca de três, cinco e sete vezes nas regiões Norte, Sudeste e Sul, respectivamente.

No estudo de conscritos de 2016, em análise multivariada realizada por Motta et al. 8, a sífilis esteve associada a fatores tais como autorrelato de antecedentes de IST pelo menos uma vez na vida $(\mathrm{OR}$ = 7,24; IC95\%: 3,99-13,13; p < 0,001), identificação como HSH (OR = 3,43; IC95\%: 1,73-6,79; p = 0,001) e primeira relação sexual antes dos 15 anos de idade (OR = 2,62; IC95\%: 1,36-5,05; p = 0,004).

É possível aproximar os dados do estudo brasileiro de pesquisa realizada nos Estados Unidos 11, que mostrou maior incidência do agravo entre homens pretos jovens, na faixa etária de 20 a 29 anos. August et al. 12 identificaram que, entre os homens mais jovens (18-30 anos), os brasileiros têm sete vezes mais chances de obter resultado reagente para uma IST (herpes genital, clamídia, gonorreia e/ ou sífilis) quando comparados aos norte-americanos. 
No presente estudo, a prevalência de sífilis foi maior entre jovens sem acesso à internet no domicílio. A Internet é uma ferramenta que pode contribuir na construção de projetos educacionais para a promoção da saúde, assim como apoiar o desenvolvimento de habilidades pessoais e sociais em práticas preventivas. Entretanto, é crucial considerar a necessidade e a viabilidade de implementação desses projetos em cada local, além das desigualdades socioculturais e econômicos nos diferentes contextos 13. Apesar de ser um instrumento de disseminação de informações úteis na área da saúde, também pode ser fonte de conteúdos falsos e de díficil distinção sobre o que de fato é confiável 14.

Aproximadamente $16 \%$ dos conscritos iniciaram a vida sexual com menos de 14 anos e também registraram maior prevalência de sífilis, em contraponto àqueles com uma iniciação mais tardia. August et al. 12 mostraram que, dos 3.047 participantes de seu estudo, a maior parte $(43,8 \%)$ iniciou a atividade sexual entre 15 e 17 anos. Aqueles cuja iniciação sexual ocorreu aos 14 anos ou antes tiveram duas vezes mais chances de apresentar teste reagente para uma IST.

A prevalência de sífilis na vida foi de 5,2\% entre os conscritos HSH, sendo cerca de duas vezes maior que a do estudo de conscritos de 2007 8. Uma pesquisa realizada em Nova Iorque (Estados Unidos) 15 e uma revisão sistemática de estudos na América Latina e no Caribe 16 revelaram aumento da prevalência de sífilis nesse segmento.

Aqueles que receberam presentes, drogas ou outros incentivos em troca de sexo se destacaram com maior prevalência de sífilis no presente inquérito, em concordância com Grillo et al. 17, que mostraram associação entre o sexo comercial $(\mathrm{ORa}=1,64$; IC95\%: 1,19-2,27) e o desfecho de sífilis e/ou HIV.

Ressalta-se também que cerca de $41 \%$ dos conscritos declararam mais de cinco parcerias sexuais no mês anterior à pesquisa e apresentaram maior prevalência de sífilis. O uso do preservativo, a testagem e demais medidas de prevenção, entre outros fatores, precisam incluir estratégias de gestão de risco para diminuir a vulnerabilidade dos jovens 18 .

Um estudo entre universitários na Nova Zelândia 19 mostrou que os estudantes se tornavam mais propensos a realizar testes de rotina para a detecção desses agravos quando passavam a conhecer as IST. Esse dado reforça a importância da educação sexual nos cenários escolares para consolidar o entendimento sobre prevenção das IST ${ }^{19}$. No presente estudo, a televisão, a Internet e a escola foram as mais relatadas fontes de informação por meio das quais os participantes gostariam de acessar conhecimentos sobre IST, coincidindo com outro achado na literatura 20.

Apesar de ser oportuno melhorar o conhecimento individual sobre questões de saúde sexual, a mudança de comportamento não é suficiente para a prevenção das IST. Outras contingências sociais e programáticas afetam o conjunto de elementos que interferem nas práticas sexuais e no acesso a cuidados 21 .

Entre os participantes que referiram história prévia de úlcera anogenital, a prevalência de sífilis foi maior, quando comparada com outros sinais e sintomas. No entanto, deve-se considerar o quadro herpético nesses conscritos. O estudo de Araújo et al. 22 mostrou associação entre ter úlcera genital e resultado reagente para sífilis, ao passo que Naveca et al. 23 encontraram prevalências de 55,3\%, 3,2\% e $8,1 \%$, respectivamente, para vírus herpes simples tipo 2 , tipo 1 e Treponema pallidum em pesquisa sobre etiologia de úlceras genitais.

Antecedentes de IST foram relatados por $20 \%$ dos jovens que apresentaram prevalência de sífilis $(2,7 \%)$, sendo que o tratamento foi realizado por apenas um terço destes jovens. $\mathrm{O}$ acesso às ações $\mathrm{e}$ serviços de saúde vem acompanhado de outros desafios, como sentimentos de vergonha, medo do estigma, barreiras socioculturais e religiosas, além de despreparo de profissionais de saúde na abordagem da sexualidade 24 .

O algoritmo utilizado é de abordagem reversa para diagnóstico de sífilis, em que se inicia o fluxograma com o teste treponêmico automatizado 25,26, como o ensaio Architect Syphilis TP, que permite a análise de muitas amostras e reduz o tempo de resposta. Nos estudos analisados pela revisão sistemática, a sensibilidade e a especificidade do Architect Syphilis TP variaram de 97,3-100\% e de 94,5-100\%, respectivamente 27 . O teste não treponêmico é o VDRL cuja sensibilidade na sífilis secundária é de $100 \%$, na sífilis primária varia de $74-87 \%$, na sífilis latente de $71-100 \%$ e na sífilis tardia de $37-94 \%$, ao passo que a especificidade é de $98 \%$. O terceiro teste é o FTA-Abs cuja sensibilidade na sífilis primária varia de $93-100 \%$, na sífilis secundária e latente é de 100\%, na sífilis terciária é de 96\%, ao mesmo tempo em que a especificidade é de $98 \% 25$. 
Enquanto não estiverem disponíveis testes com maior acurácia para o diagnóstico, é pertinente que futuros estudos contemplem o algoritmo com dois testes, utilizando o terceiro em caso de discordância dos primeiros resultados. Zoni et al. 16, em revisão sistemática, verificaram heterogeneidade dos algoritmos para identificar a prevalência de sífilis. Dos 95 estudos incluídos na pesquisa, 75\% relataram a utilização de fluxogramas com testes para a confirmação de sífilis.

Apesar de importante, a análise dos resultados de 2016 pode não refletir a situação atual. Levandose em conta a transversalidade do presente estudo, constata-se a limitação para estabelecer relações causais entre as variáveis analisadas. Também não se pode excluir a influência do viés de memória para respostas a determinadas questões que remetem a fatos ocorridos antes da pesquisa. Ainda, é possível que algumas das questões do instrumento de coleta de dados não tenham sido compreendidas adequadamente pelos respondentes.

Outra potencial limitação da pesquisa foi a exclusão de conscritos analfabetos, que foram convocados e dispensados do serviço militar ${ }^{9}$. Entretanto, as limitações da pesquisa são minimizadas no desenho do estudo transversal realizado com instrumentos de coleta de dados padronizados e medições laboratoriais, considerando o tamanho amostral muito grande 8,9 .

Mediante a garantia do sigilo e do anonimato, o caráter autoaplicado do questionário busca diminuir o viés de desejabilidade social, sobretudo nas respostas a temas sensíveis, como práticas sexuais. Mesmo sendo sigilosa a coleta de dados, o percentual de HSH pode estar subestimado. A circunstância do autorrelato pode ter levado a perdas devido a respostas inadequadas sobre informações relevantes. Embora tenha sido feito um esforço para incentivar a honestidade, comportamentos de risco relacionados às IST podem ter sido subnotificados.

Em sentido amplo, Ullrich et al. 28 observaram que gays vivendo com HIV com tendência a esconder a homossexualidade podem ter a autoestima prejudicada e, consequentemente, ter seu sistema imunológico comprometido com a diminuição da contagem de linfócitos T-CD4+. Na pesquisa com lésbicas, gays, bissexuais e transgêneros veteranos no contexto das Forças Armadas norte-americanas, Moradi 29 destacou potenciais benefícios para a harmonia no âmbito militar quando revelada a orientação sexual.

Apesar de não ter relação com o meio militar, diante do estigma, do preconceito e da dificuldade de acessar os HSH, Ezoe et al. 30 utilizaram um método específico de dimensionamento de redes sociais para estimar o tamanho desta população no Japão, de forma a facilitar a verificação de tendências epidemiológicas, o planejamento e a implementação de intervençoes eficazes na área da saúde pública.

\section{Conclusão}

A obrigatoriedade do alistamento militar e da apresentação às comissões de seleção permite a conformação de um marco amostral propício à realização de inquéritos seriados. O aumento de sífilis entre os jovens conscritos brasileiros evidenciado no presente estudo, em comparação aos inquéritos anteriores, reforça a importância desta população sentinela para realizar vigilância ativa e monitoramento de eventos em saúde pública, de forma a subsidiar estratégias de atenção à saúde dos jovens.

Convém que tais estratégias sejam integradas às políticas públicas de educação, especialmente quando se trata de jovens que estão no Ensino Médio ou em fase de conclusão deste nível de ensino. Como a escola constitui locus privilegiado para ações de promoção da saúde sexual 31, torna-se necessário e oportuno ampliar o diálogo sobre sexualidade e medidas preventivas nestes espaços. 


\section{Colaboradores}

F. L. S. Freitas, X. P. D. Bermúdez e E. Merchan-Hamann contribuíram com o desenho do estudo, revisão da literatura, análise e interpretação dos dados e redação do artigo. L. R. Motta e M. P. Paganella contribuíram com o desenho, coleta de dados e revisão do artigo. R. D. Sperhacke contribuiu com a revisão do artigo. G. F. M. Pereira contribuiu com o desenho do estudo e interpretação e discussão.

\section{Informações adicionais}

ORCID: Francisca Lidiane Sampaio Freitas (00000003-1344-6389); Ximena Pamela Díaz Bermúdez (0000-0002-3771-7684); Edgar Merchan-Hamann (0000-0001-6775-9466); Leonardo Rapone da Motta (0000-0003-3673-2687); Machline Paim Paganella (0000-0002-0829-2517); Rosa Dea Sperhacke (0000-0001-8822-4583); Gerson Fernando Mendes Pereira (0000-0001-8886-1662).

\section{Agradecimentos}

Ao Departamento de Doenças de Condições Crônicas e Infecções Sexualmente Transmissíveis, Ministério da Saúde, pelo financiamento e apoio técnico para a realização do inquérito epidemiológico; ao Ministério da Defesa, pelo apoio logístico; ao Laboratório de Pesquisa em HIV/Aids da Universidade de Caxias do Sul, pela condução da pesquisa original; e aos jovens conscritos, pela participação no estudo.

\section{Referências}

1. Hook EW. Syphilis. Lancet 2017; 389:1550-7.

2. Peeling RW, Mabey D, Kamb ML, Chen XS, Radolf JD, Benzaken AS. Syphilis. Nat Rev Dis Primers 2017; 3:17073.

3. Ministério da Saúde. Boletim epidemiológico: sífilis. Brasília: Ministério da Saúde; 2019.

4. Szwarcwald CL, Carvalho MF, Barbosa Júnior A, Barreira D, Speranza FAB, Castilho EA. Temporal trends of HIV-related risk behavior among Brazilian military conscripts, 19972002. Clinics 2005; 60:367-74.

5. Sawyer SM, Afifi RA, Bearinger LH, Blakemore S-J, Dick B, Ezeh AC, et al. Adolescence: a foundation for future health. Lancet 2012; 379:1630-40.

6. Szwarcwald CL, Andrade CLT, Pascom ARP, Fazito E, Pereira GFM, Penha IT. HIV-related risky practices among Brazilian young men, 2007. Cad Saúde Pública 2011; 27 Suppl 1:S19-26.

7. Ribeiro D, Rezende EF, Pinto VM, Mendes Pereira GF, Miranda AE. Prevalence of and risk factors for syphilis in Brazilian armed forces conscripts. Sex Transm Infect 2012; 88:32-4.

8. Motta LR, Sperhacke RD, Adami AG, Kato SK, Vanni AC, Paganella MP, et al. Syphilis prevalence and risk factors among young men presenting to the Brazilian Army in 2016. Medicine (Baltimore) 2018; 97:e13309.

9. Sperhacke RD, Motta LR, Kato SK, Vanni AC, Paganella MP, Oliveira MCP, et al. HIV prevalence and sexual behavior among young male conscripts in the Brazilian army, 2016. Medicine (Baltimore) 2018; 97:S25-31.

10. Pessoa DGC, Silva PL. Análise de dados amostrais complexos. São Paulo: Associação Brasileira de Estatística; 1998.

11. Clark LL, Hunt DJ. Incidence of syphilis, active component, U.S. Armed Forces. MSMR 2015; 22:8-16.

12. August EM, Daley E, Kromrey J, Baldwin J, Romero-Daza N, Salmeron J, et al. Age-related variation in sexual behaviors among heterosexual men residing in Brazil, Mexico and the USA. J Fam Plan Reprod Health Care 2014; 40:261-9.

13. Garbin HBR, Guilam MCR, Pereira Neto AF. Internet na promoção da saúde: um instrumento para o desenvolvimento de habilidades pessoais e sociais. Physis (Rio J.) 2012; 22:347363.

14. Gottlieb S. Health information on internet is often unreliable. BMJ 2000; 321:136.

15. Schillinger JA, Slutsker JS, Pathela P, Klingler E, Hennessy RR, Toro B, et al. The epidemiology of syphilis in New York City: historic trends and the current outbreak among men who have sex with men, 2016. Sex Transm Dis 2018; 45(9S Suppl 1):S48-54.

16. Zoni AC, Nica M, González A, Werin H, Gren S. Syphilis in the most at-risk populations in Latin America and the Caribbean: a systematic review. Int J Infect Dis 2013; 17:e84-92. 
17. Grillo M, Tran BR, Tamoufe U, Djoko CF, Saylors K, Woodland K, et al. HIV and syphilis prevalence and associated risks in the Cameroonian Armed Forces. Curr HIV Res 2017; 15:137-45.

18. Slater C, Robinson AJ. Sexual health in adolescents. Clin Dermatol 2014; 32:189-95.

19. Denison HJ, Bromhead C, Grainger R, Dennison EM, Jutel A. What influences university students to seek sexually transmitted infection testing? A qualitative study in New Zealand. Sexual \& Reproductive Healthcare 2018; 16:56-60.

20. Tilson EC, Sanchez V, Ford CL, Smurzynski M, Leone PA, Fox KK, et al. Barriers to asymptomatic screening and other STD services for adolescents and young adults: focus group discussions. BMC Public Health 2004; 4:21.

21. Lorimer K, McMillan L, McDaid L, Milne D, Russell S, Hunt K. Exploring masculinities, sexual health and wellbeing across areas of high deprivation in Scotland: the depth of the challenge to improve understandings and practices. Health Place 2018; 50:27-41.

22. Araújo MAL, Rocha AFB, Cavalcante EGF, Moura HJ, Galvão MTG, Lopes ACMU. Doenças sexualmente transmissíveis atendidas em unidade primária de saúde no Nordeste do Brasil. Cad Saúde Colet (Rio J.) 2015; 23:347-53.

23. Naveca FG, Sabidó M, Almeida TAP, Veras EA, Mejía MCC, Galban E, et al. Etiology of genital ulcer disease in a sexually transmitted infection reference center in Manaus, Brazilian Amazon. PLoS One 2013; 8:e63953.
24. Newton-Levinson A, Leichliter JS, ChandraMouli V. Sexually transmitted infection services for adolescents and youth in low- and middle-income countries: perceived and experienced barriers to accessing care. J Adolesc Health 2016; 59:7-16.

25. Organização Mundial da Saúde. Diagnóstico laboratorial de doenças sexualmente transmissíveis, incluindo o vírus da imunodeficiência humana. Brasília: Ministério da Saúde; 2015.

26. Departamento de Vigilância, Prevenção e Controle das Infecções Sexualmente Transmissíveis, do HIV/Aids e das Hepatites Virais, Secretaria de Vigilância em Saúde, Ministério da Saúde. Manual técnico para diagnóstico da sífilis. Brasília: Ministério da Saúde; 2016.

27. Park IU, Tran A, Pereira L, Fakile Y. Sensitivity and specificity of treponemal-specific tests for the diagnosis of syphilis. Clin Infect Dis 2020; 71 Suppl 1:S13-20.

28. Ullrich PM, Lutgendorf SK, Stapleton JT, Horowitz M. Self regard and concealment of homosexuality as predictors of CD4+ cell count over time among HIV seropositive gay men. Psychol Health 2004; 19:183-96.

29. Moradi B. Sexual orientation disclosure, concealment, harassment, and military cohesion: perceptions of LGBT military veterans. Mil Psychol 2009; 21:513-33.

30. Ezoe S, Morooka T, Noda T, Sabin ML, Koike S. Population size estimation of men who have sex with men through the network scale-up method in Japan. PLoS One 2012; 7 :e31184.

31. Ministério da Saúde. Proteger e cuidar da saúde de adolescentes na atenção básica. Brasília: Ministério da Saúde; 2017. 
Abstract

The objective was to characterize sociodemographic, behavioral, and clinical aspects in young Brazilian military recruits according to prevalence of syphilis. This was a descriptive study based on a nationwide population-based survey in 2016 with a probabilistic sample of military recruits 17 to 22 years of age. A confidential self-applied questionnaire was used. Blood samples were drawn from participants for treponemal and non-treponemal syphilis tests. Descriptive statistical techniques were used to estimate syphilis prevalence rates and distribution of frequencies between the target variables, considering 95\% confidence intervals (95\%CI), after weighting the data. Of the total of 37,282 participants, $73.7 \%$ were sexually initiated. Prevalence rates for lifetime and confirmed syphilis were $1.6 \%$ and $1.1 \%$, respectively. The following population variables showed higher prevalence of syphilis: lack of Internet access at home; initiation of sexual activity before 14 years of age; men who have sex with men; more than five sexual partners; having received presents, drugs, or other incentives in exchange for sex; and prior history of symptoms of sexually transmissible infections. An increase was observed in syphilis in Brazilian military recruits when compared to previous surveys. This increase emphasizes the importance of this sentinel population for performing active surveillance in order to support healthcare strategies for youth, including in the school system.

Prevalence; Health Surveys; Syphilis

\section{Resumen}

El objetivo fue caracterizar aspectos sociodemográficos, comportamentales y clínicos entre reclutas brasileños, según la prevalencia de sífilis. Se trata de un estudio descriptivo, desarrollado a partir de una investigación nacional de base poblacional, realizada en 2016, con una muestra probabilística de jóvenes reclutas de 17 a 22 años de edad. Se realizó una autoaplicación de cuestionarios confidenciales. Las muestras de sangre total de los participantes fueron recogidas para la prueba de sífilis, con la utilización de pruebas treponémicas y no treponémicas. Se emplearon técnicas de estadística descriptiva para estimar las prevalencias de sifilis y distribución de frecuencias entre las variables investigadas, considerando intervalos del 95\% de confianza (IC95\%), tras la ponderación de los datos. Del total de 37.282 participantes, un 73,7\% habian iniciado su vida sexual. Las prevalencias de sifilis en la vida y de sifilis confirmada fueron de $1,6 \%$ y 1,1\%, respectivamente. Las siguientes variables poblacionales presentaron una mayor prevalencia de sífilis: ausencia de acceso a Internet en el domicilio; inicio de la actividad sexual antes de los 14 años; categoría de exposición hombres que practican sexo con hombres; prácticas sexuales con más de cinco personas; recibimiento de regalos, drogas u otros incentivos a cambio de sexo; e historia previa de sintomatología de infecciones sexualmente transmisibles. Se observó el aumento de sifilis entre los jóvenes reclutas brasileños, en comparación con las encuestas anteriores. Tal incremento refuerza la importancia de esa población centinela para realizar una vigilancia activa, de forma que se apoyen estrategias de atención a la salud de los jóvenes, incluyendo escenarios escolares.

Prevalencia; Encuestas Epidemiológicas; Sifilis

Recebido em 05/Set/2020

Versão final reapresentada em 30/Nov/2020

Aprovado em 28/Dez/2020 